\title{
Hymenoptera Venom Immunotherapy: How to Safely Switch to the Same Venom From a Different Manufacturer
}

Bilò $\mathrm{MB}^{1}$, Martini $\mathrm{M}^{1}$, Berra $\mathrm{D}^{2}$, Scarpa $\mathrm{A}^{3}$, Losappio $\mathrm{L}^{4}$, Quercia $\mathrm{O}^{5}$, Lodi Rizzini $\mathrm{F}^{6}$, Bignardi $\mathrm{D}^{7}$, Cortellini $\mathrm{G}^{8}$, Zisa $\mathrm{G}^{9}$, Del Giudice $\mathrm{A}^{10}$, Manzotti $\mathrm{G}^{11}$, Marcotulli $\mathrm{C}^{12}$, Murzilli $\mathrm{F}^{13}$, Cilia $\mathrm{M}^{14}$, Pravettoni $\mathrm{V}^{15}$, Borrelli $\mathrm{P}^{16}$, Pastorello $\mathrm{EA}^{4}$

${ }^{1}$ Allergy Unit, Department of Internal Medicine, University Hospital of Ancona - Allergy and Clinical Immunology School, Università Politecnica delle Marche, Ancona, Italy

${ }^{2}$ Allergology Service, Pneumology Unit, Busto Arsizio Hospital, Varese, Italy

${ }^{3}$ Allergy Unit - Dermatology, Department of Surgery, Hospital of Mirano, Mirano, Italy

${ }^{4}$ Department of Allergy and Immunology, Asst Grande Ospedale Metropolitano Niguarda, University of Milano, Italy

${ }^{5}$ Allergology High Specialty Unit General Medicine, Faenza Hospital Ravenna, Ravenna, Italy

${ }^{6}$ Allergy Unit, Spedali Civili Brescia, Department of Clinical and Experimental Sciences, University of Brescia, Brescia, Italy ${ }^{7}$ Allergy Unit, Department of Internal Medicine, Ospedale Policlinico San Martino of Genoa, Genoa, Italy

${ }^{8}$ Internal Medicine and Rheumatology Unit, Rimini Hospital, Rimini, Italy

${ }^{9}$ Allergy Unit, Department of Internal Medicine, Azienda Ospedaliero-Universitaria Maggiore della Carità, Novara, Italy ${ }^{10}$ Allergy Unit, Department of Internal Medicine, Hospital Martini of Turin, Turin, Italy

${ }^{11}$ Allergy Unit, Department of Internal Medicine, Treviglio General Hospital, ASST Bergamo Ovest, Treviglio, Italy

${ }^{12}$ Allergy Unit, Department of Pneumology, Asst Garda, Desenzano, Italy

${ }^{13}$ Allergy Unit, Department of Internal Medicine, S.S. Filippo and Nicola Hospital of Avezzano, Azienda Sanitaria Locale n. 1 dell' Abruzzo, Avezzano, Italy

${ }^{14}$ Allergy Unit, Health Care Service, Scilla, Italy

${ }^{15}$ UOC General Medicine - Immunology and Allergology - IRCCS Foundation Ca'Granda Ospedale Maggiore Policlinico, Milan, Italy ${ }^{16}$ Allergy Unit, Department of Internal Medicine, U. Parini Regional Hospital, Aosta, Italy

J Investig Allergol Clin Immunol 2018; Vol. 28(3): 205-208 doi: $10.18176 /$ jiaci.0242

Key words: Extract switching. Hymenoptera. Immunotherapy. Mastocytosis. Safety.

Palabras clave: Cambio de extracto. Himenóptero. Inmunoterapia. Mastocitosis. Seguridad. 
The literature provides no clear approach to VIT-treated patients if the allergenic extract is no longer available $[1,2,4]$. However, for safety reasons, current clinical practice discourages abrupt switching to the same venom extract from a different manufacturer without an induction phase.

In early 2016, a national shortage of aqueous VIT preparations led clinicians to switch to another extract based on their experience.

We prospectively collected data on VIT switching from 16 Italian centers with broad experience in management of VIT. Whenever discontinuation was not recommended, patients switched to a preparation of the same venom made by another manufacturer. Before VIT, all patients signed an informed consent form and underwent a medical examination. A venous access was also placed. The baseline characteristics of the patients are shown in the Table.
Of the 531 cases recorded in 523 patients ( 8 patients underwent double VIT), 349 cases (Group A, 66\%) switched to a new extract on a single day: their own maintenance dose, which was reached entirely with the new extract, was divided into 3 aliquots in 276 cases and 2 aliquots in 73 cases, with a 30-45 minute interval between injections. In 95 cases (Group $\mathrm{B}, 18 \%$ ), the switch was performed on a single day by dividing the maintenance dose into 2 different aliquots injected at a 30-45 minute interval; however, only the old extract was used for the first injection (30\% and $50 \%$ of the total maintenance dose in 31 and 64 cases, respectively), whereas at the second injection, only the new extract was injected. All the other switches $(n=87)$ involved dose reduction by injecting different amounts of the new extract on the first day (ranging from $10 \%$ to $80 \%$ of the maintenance dose) and then gradual increases during subsequent visits until $100 \%$ of the maintenance

Table. Characteristics of Patients and VIT Switching

\begin{tabular}{|c|c|c|c|c|}
\hline & Total & Group A & Group B & Group C \\
\hline Patients, No. & 523 & $342(65.39 \%)$ & $94(17.97 \%)$ & $87(16.63 \%)$ \\
\hline Male/Female & $391 / 132$ & $258 / 84$ & $70 / 24$ & $63 / 24$ \\
\hline Mean age (range), $y$ & $55.5(8-90)$ & $55.5(11-90)$ & $54.6(8-85)$ & $48.9(11-82)$ \\
\hline $\begin{array}{l}\text { Reaction type }^{\mathrm{a}} \\
\text { Grade I } \\
\text { Grade II } \\
\text { Grade III } \\
\text { Grade IV }\end{array}$ & $\begin{array}{c}38(7.27 \%) \\
70(13.38 \%) \\
185(35.37 \%) \\
230(43.98 \%)\end{array}$ & $\begin{array}{c}15(39.47 \%) \\
40(57.14 \%) \\
128(36.68 \%) \\
166(70.64 \%)\end{array}$ & $\begin{array}{l}14(36.84 \%) \\
13(18.57 \%) \\
31(16.49 \%) \\
37(15.74 \%)\end{array}$ & $\begin{array}{c}9(23.68 \%) \\
17(24.29 \%) \\
29(15.43 \%) \\
32(13.62 \%)\end{array}$ \\
\hline Mastocytosis and/or high bSTC & $62(11.68 \%)$ & $44(70.97 \%)$ & $11(17.74 \%)$ & $7(11.29 \%)$ \\
\hline Patients with double VIT, No. & 8 & 7 & 1 & 0 \\
\hline $\begin{array}{l}\text { Type of VIT } \\
\text { Apis mellifera } \\
\text { Polistes species/P dominula } \\
\text { Vespula } \text { species } \\
\text { Vespa crabro }\end{array}$ & $\begin{array}{c}58(10.92 \%) \\
83(15.63 \%) \\
389(73.26 \%) \\
1(0.19 \%)\end{array}$ & $\begin{array}{c}36(62.07 \%) \\
54(65.06 \%) \\
258(66.32 \%) \\
1(100 \%)\end{array}$ & $\begin{array}{c}12(20.69 \%) \\
9(10.84 \%) \\
74(19.02 \%) \\
0(0.00 \%)\end{array}$ & $\begin{array}{c}10(17.24 \%) \\
20(24.10 \%) \\
57(14.65 \%) \\
0(0.00 \%)\end{array}$ \\
\hline $\begin{array}{l}\text { Mean (SD) administration interval, wk } \\
\text { Mean (SD) VIT period, y }\end{array}$ & $\begin{array}{l}8(2.6) \\
7(5.6)\end{array}$ & & & \\
\hline Switches, No. & 531 & $349(65.73 \%)$ & $95(17.89 \%)$ & $87(16.38 \%)$ \\
\hline Switch protocol & / & $\begin{array}{l}\text { Full dose } \\
1 \text { day }\end{array}$ & $\begin{array}{l}\text { Full dose } \\
1 \text { day }\end{array}$ & $\begin{array}{l}\text { Reduced dose } \\
1 \text { day }^{\mathrm{c}}\end{array}$ \\
\hline Extract & / & $\mathrm{N}$ & $\mathrm{N}+\mathrm{O}^{\mathrm{d}}$ & $\mathrm{N}$ \\
\hline \multicolumn{5}{|l|}{ Type of new extract } \\
\hline Aqueous & $357(67.23 \%)$ & $227(63.59 \%)$ & $64(17.93 \%)$ & $66(18.49 \%)$ \\
\hline $\operatorname{Depot}^{f}$ & $174(32.77 \%)$ & $122(70.11 \%)$ & $31(17.82 \%)$ & $21(12.07 \%)$ \\
\hline Systemic reaction & $1(0.19 \%)$ & 0 & 0 & 1 \\
\hline
\end{tabular}

Abbreviations: N, new extract; O, old extract; bSTC, serum baseline tryptase concentration; VIT, venom immunotherapy.

${ }^{a}$ Mueller grading system [6].

${ }^{b}$ Forty-four patients (46\%) were treated with mixed American Polistes extract (Polistes annularis, fuscatus, and exclamans species), and all the others were treated with Polistes dominula extracts.

${ }^{\mathrm{c}} 100 \%$ of new extract was used during the following visits.

${ }^{\mathrm{d}}$ The dose was gradually increased to the full dose during the following visits.

ePurified, nonpurified, or capillary extracted extracts.

fPurified aluminium hydroxide adsorbed or tyrosine adsorbed extracts. 
dose was reached (Group C, 16\%). The extracts used were both aqueous (purified aqueous, 10\%; nonpurified aqueous, $51 \%$; capillary extracted aqueous, 6\%) and depot (purified aluminium hydroxide adsorbed, 16\%; tyrosine adsorbed, $17 \%$ ). All the Polistes-allergic patients were switched to the new Polistes dominula extracts. The switch protocols and the extracts used are reported in the Table. Subsequent administration of maintenance VIT doses was scheduled with a timeframe similar to that followed before the switch (8 [2.6] weeks).

The switch was well tolerated in all but 1 patient. One grade I systemic reaction (SR) [5] was reported in an 82-year-old man who had been receiving $100 \mu \mathrm{g}$ of Vespula VIT for 3.8 years (Group C). The patient had mastocytosis and ischemic heart disease treated with a $\beta$-blocker and a sartan. He experienced generalized pruritus a few hours after the injection $(60 \mu \mathrm{g})$ and recovered spontaneously in 4 days. His nonpurified Vespula aqueous extract was switched to a purified aluminium hydroxide adsorbed preparation. He tolerated subsequent injections at the same total dose after 1 week and at a dose of $100 \mu \mathrm{g}$ after a further week, with no SRs. Local reactions were not taken into consideration, as they are very common.

No SRs were reported in the other patients with mastocytosis and/or elevated serum baseline tryptase concentration $(n=61)$, even though $44(71 \%)$ followed the Group A protocol. Similarly, SRs were not observed in patients with cardiovascular comorbidity (19\% of patients) or respiratory comorbidity $(3 \%)$ or in patients treated with ß-blockers (4\%), ACE-inhibitors (5\%), or sartans (9\%). In addition, adverse effects were not reported in any bee venom-allergic patients $(n=58)$ or in patients with a long interval between injections (more than 8 weeks) (35\%) before switching. Moreover, patients previously treated with a mix of American Polistes venom tolerated the switch to $P$ dominula extracts. None of the patients reported any subsequent SRs during a mean follow-up period of 18 (4.9) months.

This is the first real-life multicenter study showing the safety of switching VIT to the same venom from another manufacturer in a large number of patients. No severe SRs were reported. No significant differences were observed between 1-day protocols (Group A and B) and the dose reduction protocols (Group C), suggesting that there is no need for an induction phase using lower doses of venom. We propose 3 explanations for our encouraging safety results. First, even though $79 \%$ of patients developed a class III-IV SR [6] at baseline and $27 \%$ had a comorbid condition, the venom was switched mainly after a maintenance phase of several years without adverse effects. Second, patients were managed by clinicians with specific expertise in VIT. Third, the comorbidities and treatments analyzed do not seem to be risk factors for severe adverse effects, individually. Interestingly, the only SR reported occurred in a mastocytosis patient with ischemic heart disease treated with a ß-blocker and a sartan. Even though the SR was mild and affected only the skin, we cannot rule out the possibility that the combination of mastocytosis with cardiovascular disease could have played a role in the case we report. In clinical practice, special attention should be paid in patients with comorbidities, especially when mastocytosis occurs with other severe comorbidities.
Although bee venom is considered a risk factor for adverse effects during VIT and different allergen compositions have been demonstrated for bee venom extracts [7], our results showed that bee venom allergy does not seem to be related to a higher risk of SRs during switching. Similarly, switching from American Polistes species to $P$ dominula venom did not cause any SRs, despite the incomplete cross-reactivity between American and European Polistes venom [8,9].

Notwithstanding the pitfalls normally associated with multicenter real-life studies, such as the different protocols and extracts used in this study, no differences in safety were observed between aqueous extracts and depot preparations or between the purified and nonpurified aqueous formulations.

Before switching, no patients in our study experienced severe SRs to the old extract, although we believe that, in this case, VIT should be restarted with a rush/ultrarush protocol in centers with broad experience in HVA and VIT [10], or with a conventional protocol in less experienced centers.

The unexpected and accidental shortage of some extracts was the only reason that made the VIT switch necessary in our study.

In conclusion, our results showed that switching VIT, if strictly necessary, is a safe option in patients who previously tolerated VIT, even without reducing the maintenance dose already reached by the patient and when performed in an appropriate environment by experienced staff.

\section{Funding}

The authors declare that no funding was received for the present study.

\section{Conflicts of Interest}

MBB has received speaker's honoraria and/or travel support from ALK-Abelló and Thermo Fisher Scientific. She has also received consultancy fees from ALK-Abelló for participation in an advisory board.

AS has received reimbursement for symposia from ALK Abelló and Stallergenes.

The other authors declare that they have no conflict of interests.

\section{References}

1. Bonifazi $F$, Jutel $M$, Bilo BM, Birnbaum J, Muller U, EAACl Interest Group on Insect Venom Hypersensitivity. Prevention and treatment of hymenoptera venom allergy: guidelines for clinical practice. Allergy. 2005;60:1459-70.

2. Sturm GJ, Varga E-M, Roberts G, Mosbech H, Bilò MB, Akdis $\mathrm{CA}$, et al. EAACl Guidelines on Allergen Immunotherapy: Hymenoptera venom allergy. Allergy. 2018;73:744-64.

3. Bonadonna P, Gonzalez-de-Olano D, Zanotti R, Riccio A, De Ferrari L, Lombardo $C$ et al. Venom immunotherapy in patients with clonal mast cell disorders: efficacy, safety, and practical considerations. J Allergy Clin Immunol Pract. 2013;1:474-8.

4. Wolf BL, Hamilton RG. Near-fatal anaphylaxis after Hymenoptera venom immunotherapy. J Allergy Clin Immunol. 1998;102:527-8. 
5. Cox L, Larenas-Linnemann D, Lockey RF, Passalacqua G. Speaking the same language: The World Allergy Organization Subcutaneous Immunotherapy Systemic Reaction Grading System. J Allergy Clin Immunol. 2010;125:569-74.

6. Mueller HL. Diagnosis and treatment of insect sensitivity. J Asthma Res. 1966;3:331-3

7. Blank S, Seismann H, Michel Y, Mclntyre M, Cifuentes L, Braren I, et al. Api m 10, a genuine A. mellifera venom allergen, is clinically relevant but underrepresented in therapeutic extracts. Allergy. 2011;66:1322-9.

8. Severino MG, Campi P, Macchia D, Manfredi M, Turillazzi S, Spadolini I, et al. European Polistes venom allergy. Allergy. 2006 Jul;:61(7):860-3.

9. Schiener M, Eberlein B, Moreno-Aguilar C, Pietsch G, Serrano $P$, Mclntyre $M$, et al. Application of recombinant antigen 5 allergens from seven allergy-relevant Hymenoptera species in diagnostics. Allergy. 2017;72:98-108.

10. Birnbaum J, Ramadour M, Magnan A, Vervloet D. Hymenoptera ultra-rush venom immunotherapy (210 min): a safety study and risk factors. Clin Exp Allergy. 2003;33:58-64.

- Manuscript received December 12, 2017; accepted for publication February 16, 2018.

Maria Beatrice Bilò

Allergy Unit, Department of Internal Medicine University Hospital of Ancona Via Conca 71

60126 Ancona, Italy

E-mail: MariaBeatrice.Bilo@ospedaliriuniti.marche.it 